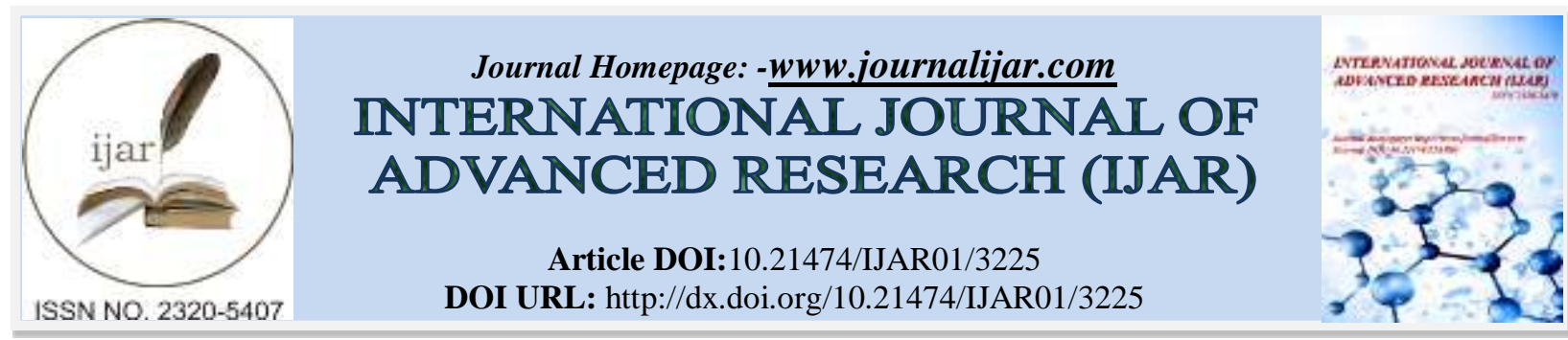

RESEARCH ARTICLE

\title{
DESTRUCTIVE DIELECTRIC CHARACTERIZATION OF MATERIAL USING MICROSTRIP RING RESONATOR.
}

\author{
Hussein Kassem ${ }^{1}$, Hayssam El-Hajj ${ }^{1}$, Rabih Rammal ${ }^{1}$, Ismail El-Sayad ${ }^{1}$, Mohammad Hawila ${ }^{2}$ and Ahmad \\ Hamdan ${ }^{2}$.
}

1. International university of Beirut (BIU), Beirut, Lebanon.

2. Lebanese international university (LIU), Beirut, Lebanon.

\section{Manuscript Info}

Manuscript History

Received: 07 April 2018

Final Accepted: 09 May 2018

Published: June 2018

Keywords:-

Permittivity, Dielectric Constant, Thin

Films, microstrip ring resonator, monofrequency

\begin{abstract}
Material measurement is a very important step to be conducted before integrating materials in components and devices. The method used here is a mono-frequency method using Microstrip Ring resonator (MRR). The material to be measured can either be integrated within the MRR structure forming a multilayered device and thus destructive measurement or the case of the MRR sandwiched between the substrate and the material to be measured thus forming a Non-destructive measurement. The method uses the MRR as a sensor with specific dimensions and specific equation characterizing the relation between its dimensions, layer thicknesses and permittivity of the layers. The inverse problem to extract the permittivity of the unknown material is an equation based one. The results of measurement are compared to those of finite element method simulation software and the error is nonsignificant.
\end{abstract}

Copy Right, IJAR, 2018,. All rights reserved.

\section{Introduction:-}

Material Characterization is an essential procedure in the domain of materials science, without which no logical comprehension of designing materials can be found out. Several definitions terminate the term's use to techniques which examine the microscopic structure and the characteristics of materials [1,2]. Dielectric properties measurements form the scope of this paper.

Many methods to characterize materials exist such as reflection methods, free space measurement methods, transmission line, Resonators, capacitive, etc. The most familiar are Resonance methods known to be accurate than any other techniques for low loss materials.

There are two main sorts of permittivity measurements strategies: "destructive" and "Non-destructive" methods. A destructive one is performed and the material under test cannot be reused again in applications. While in Nondestructive methods, the material tested can be re-used in applications. The non-destructive techniques are less expensive to execute and simple to manufacture.

Nowadays, most electrical equipment consists of dielectric materials, each one specified by different values of permittivity and conductivity. Material measurement is a very important step to be conducted before integrating 
these materials in some applications. The method used here to extract the permittivity is Microstrip Ring Resonator [3-5]. To extract permittivity, 2 strategies are used, First the material to be measured is printed on the ring substrate (destructive strategy) and then when the ring resonator is sandwiched between 2 materials (non-destructive strategy). The measurement will be conducted at the resonant frequency.

In this work S11 and S21 parameters are extracted to find the resonant frequency and an equation based inverse problem is used to extract the permittivity of the material.

\section{System Design:-}

Every material has a special series of electrical characteristics that are dependent on its dielectric properties. So the using of these measurements are precise then it can give researchers and architects commendable data to legitimately use a material into a specific application from high speed circuits to satellite and telemetry application.

There are different methods of dielectric properties measurements like non-resonant and resonant methods [3-5]. Resonant methods do not have a sweep frequency capability for the measured frequency, but they are considered more accurate than any other techniques for low loss material and they have possible Q-factors and results in very high sensitivity. So the resonators can be used as sensors of different physical quantities which depend upon dielectric constant like complex permittivity of MUT. A ring resonator structure on a printed circuit board (PCB) can be utilized to deduce the complex permittivity $\in$ of the substrate material. The measurement rely on $\mathrm{S} 21$ parameter of a two-port ring resonator to determine the $\in$ of the board substrate.

The goal is to extract the unknown permittivity of any material using microstrip ring resonator (MRR) after making several simulations; when the ring is printed on the material (Destructive) and the ring is sandwiched between the 2 materials (Non-Destructive).

\section{System Specifications:-}

A design MRR-Ferro is designed using HFSS 3D EM software, High frequency structural simulator. It gives high performance field wave for EM field.

\section{Hardware Specifications:-}

Microstrip Ring resonator

Substrate material (low part): sapphire, $\mathrm{MgO}$

Substrate + thin film: $\mathrm{MgO}+$ Ferro

Ring and transmission lines: gold

Two parameters are varied under simulator:

Height of substrate $=$ 'e' different values from ( 0.5 to 10 um at step unit $=0.5 \mathrm{um})$

Real permittivity $=" \in$ " (different values from 50 to 350 at step unit=50)

\section{Theory of Ring Resonator:-}

This section presents a theoretical analysis for a ring resonator-based method for the estimation of complex dielectric permittivity of materials. This structure comprises of transmission lines and a ring printed on a dielectric substrate.

A ring resonator is primarily comprised of a transmission line forming a closed loop and can be implemented using Microstrip [6-8] as shown below in the fig.1. 


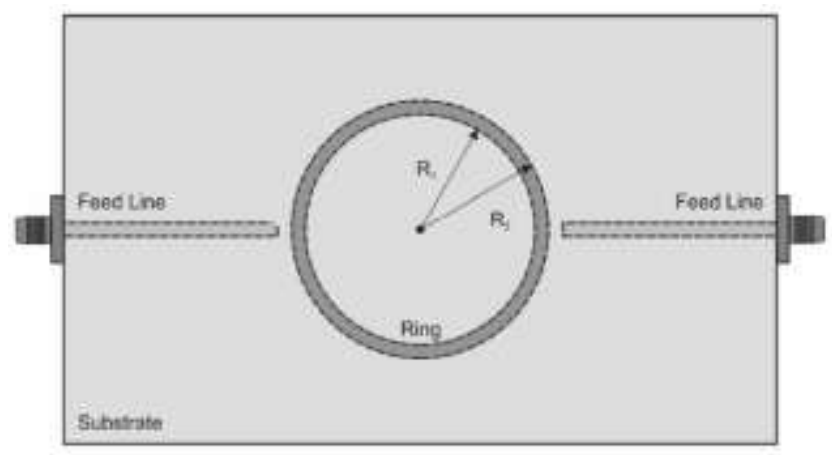

Figure 1:-Planar Ring Resonator structure.

The resonance of this resonator follows the below condition:-

$$
2 \pi \mathrm{rm} \cdot \beta=2 \mathrm{n} \pi
$$

With $\mathrm{n}=1,2,3 \ldots$ and " $\beta$ " the phase constant along the microstrip line defined by:

$$
\beta=n \pi / 1
$$

Where "l" is the resonance length related to the wave length

$$
\lambda_{\mathrm{d}}=\frac{\lambda}{\sqrt{\varepsilon_{\mathrm{eff}}}}
$$

$\mathrm{rm}$ " is the average radius of the ring and $\varepsilon_{\mathrm{eff}}$ is the effective permittivity, calculated from:

$$
\varepsilon_{\mathrm{eff}}=\frac{\varepsilon_{\mathrm{r}+1}}{2}+\frac{\varepsilon_{\mathrm{r}-1}}{2}\left(\frac{1}{1+\frac{12 \mathrm{~h}}{\mathrm{w}}}\right)
$$

To evaluate the resonance frequency of order $\mathrm{s:} \mathrm{f}_{\mathrm{r}, \mathrm{S}}$ " the following formula is used:

$$
\varepsilon_{\text {eff,s }}=\left[\mathrm{s.c} /\left(2 . \pi \mathrm{r}_{\mathrm{m}} \mathrm{f}_{\mathrm{r}, \mathrm{s}}\right)\right]^{2}
$$

s.c: radius of the gap

The width of the microstrip is chosen such that to get a characteristic impedance of $50 \Omega$, and the arm length is computed using:

\section{Coupling Gap:-}

$$
L_{s}=\frac{C}{2 f_{r} \sqrt{\varepsilon_{\text {eff }}}}
$$

So as to have a resonant frequency, a resonator can be fed by microstrip line through coupling gap. These types of coupling are called free coupling. It brings about poor return loss and transmission response, If the gap is reduced, the gap capacitance increases. Under tight coupling gap, capacitance becomes appreciable. This causes the major resonant frequency to deviate from the true value. More tightly the coupling, bigger is the deviation.

\section{Implementation/Simulation and Testing:-}

\section{Destructive:-}

The circuit without the thin film and a substrate of relative permittivity $\epsilon=10$ is shown in the fig. 2 below. The circuit is implemented and simulated under HFSS EM simulator. 


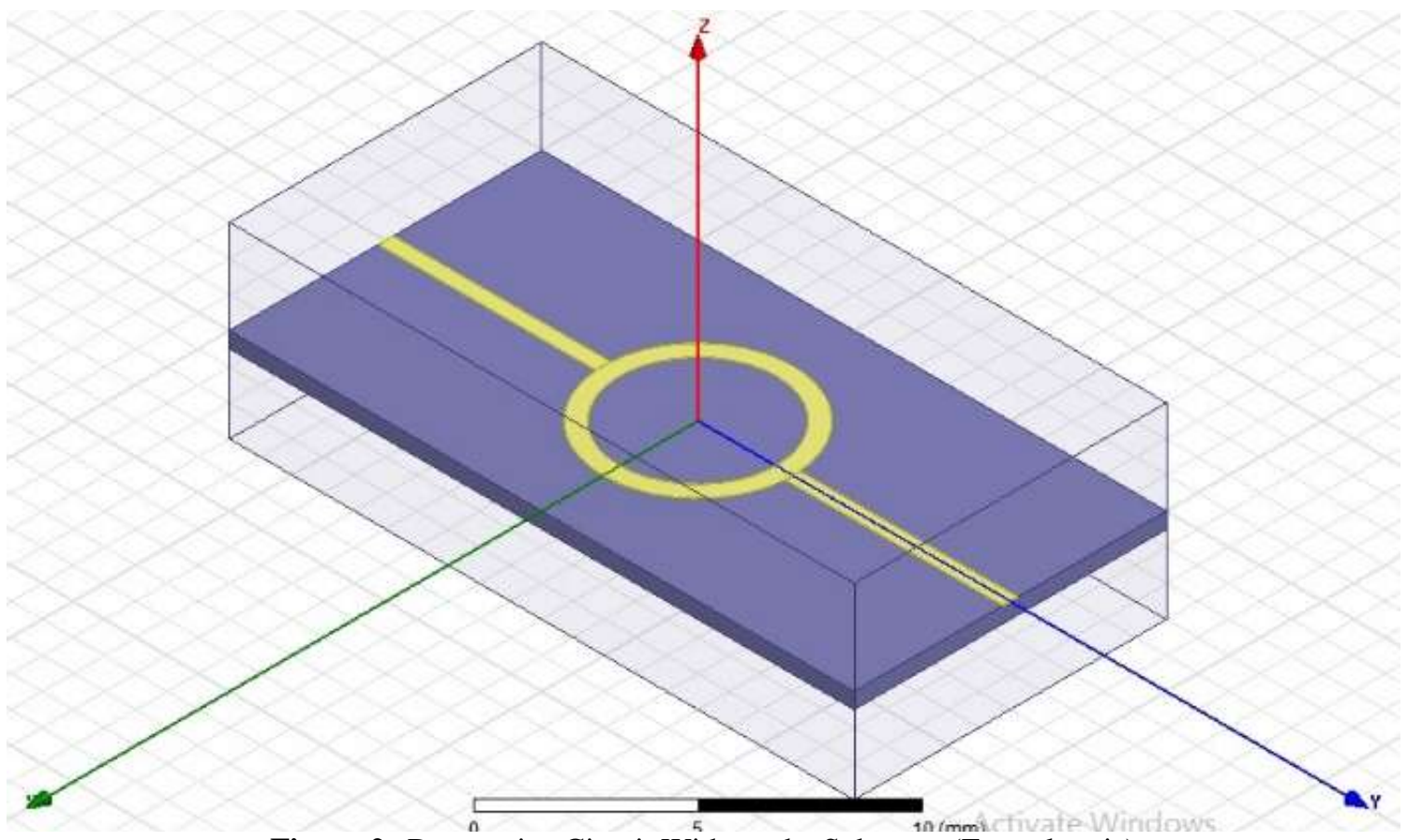

Figure 2:-Destructive Circuit Without the Substrate (Ferroelectric)

The result of simulation is displayed in the fig. 3 , where resonance appear at the following frequencies:

$$
\begin{gathered}
\mathrm{f}_{\mathrm{r}, 1}=6.5304 \mathrm{GHZ} \\
\mathrm{f}_{\mathrm{r}, 2}=12.9160 \mathrm{GHZ} \\
\mathrm{f}_{\mathrm{r}, 3}=18.8027 \mathrm{GHZ}
\end{gathered}
$$

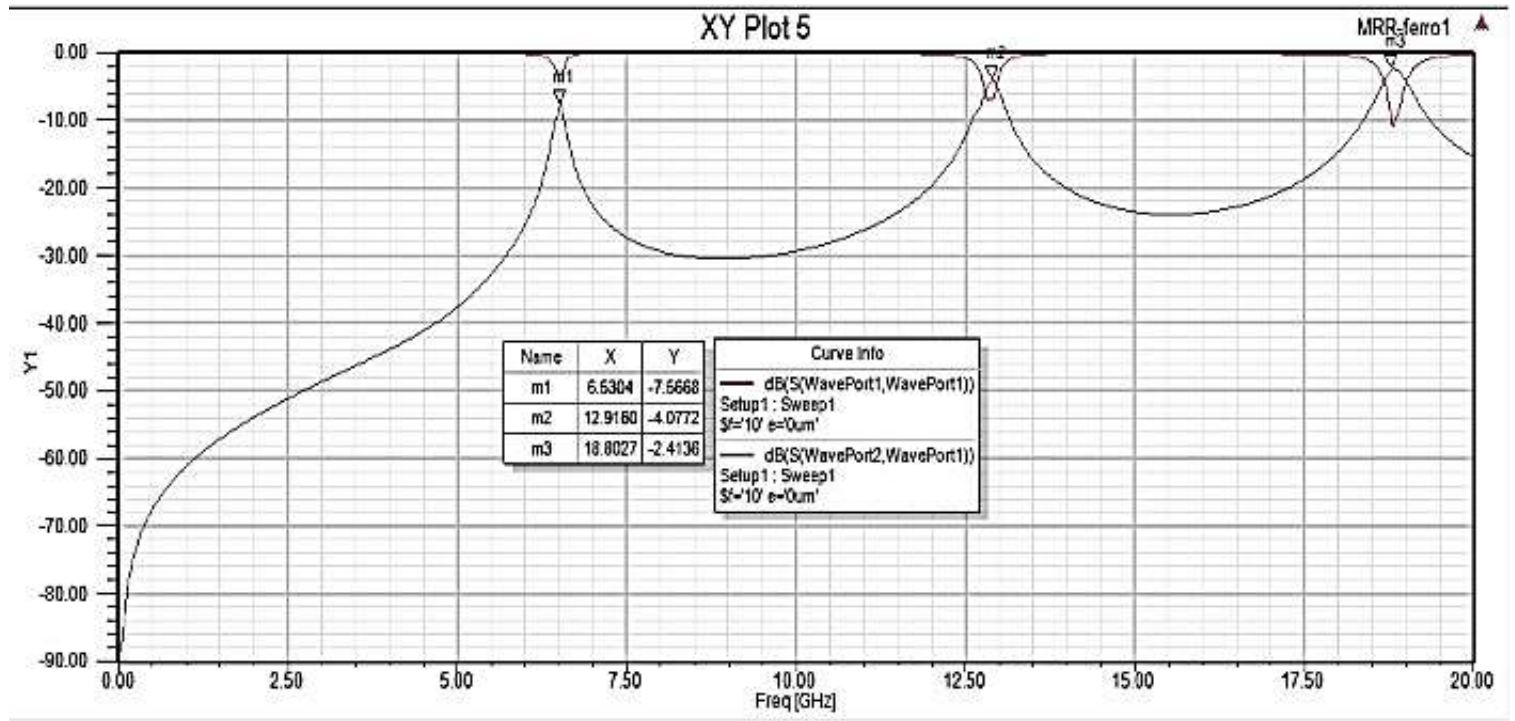

Figure 3:-Result for Destructive Circuit Without the Substrate (Ferro electric) Red Line (S11-Parameter) Black Line (S21-Parameter)

The fig. 3 shows the reflection and the transmission parameters of the circuit.

In the second step, the thin film is inserted into the circuit, and simulation is done again. The simulation is done varying the values of the film permittivity (from 50 to 350 by step unit 50) and also to check the effect of the thin film thickness, different values of thickness (from 0.5 to 10 um by step unit $0.5 \mathrm{um}$ ) are taken and simulated. 
The fig.4 below shows the additional layer added to the model in fig.2. Now, the film is sandwiched between the MRR structure and the dielectric substrate (figure3).

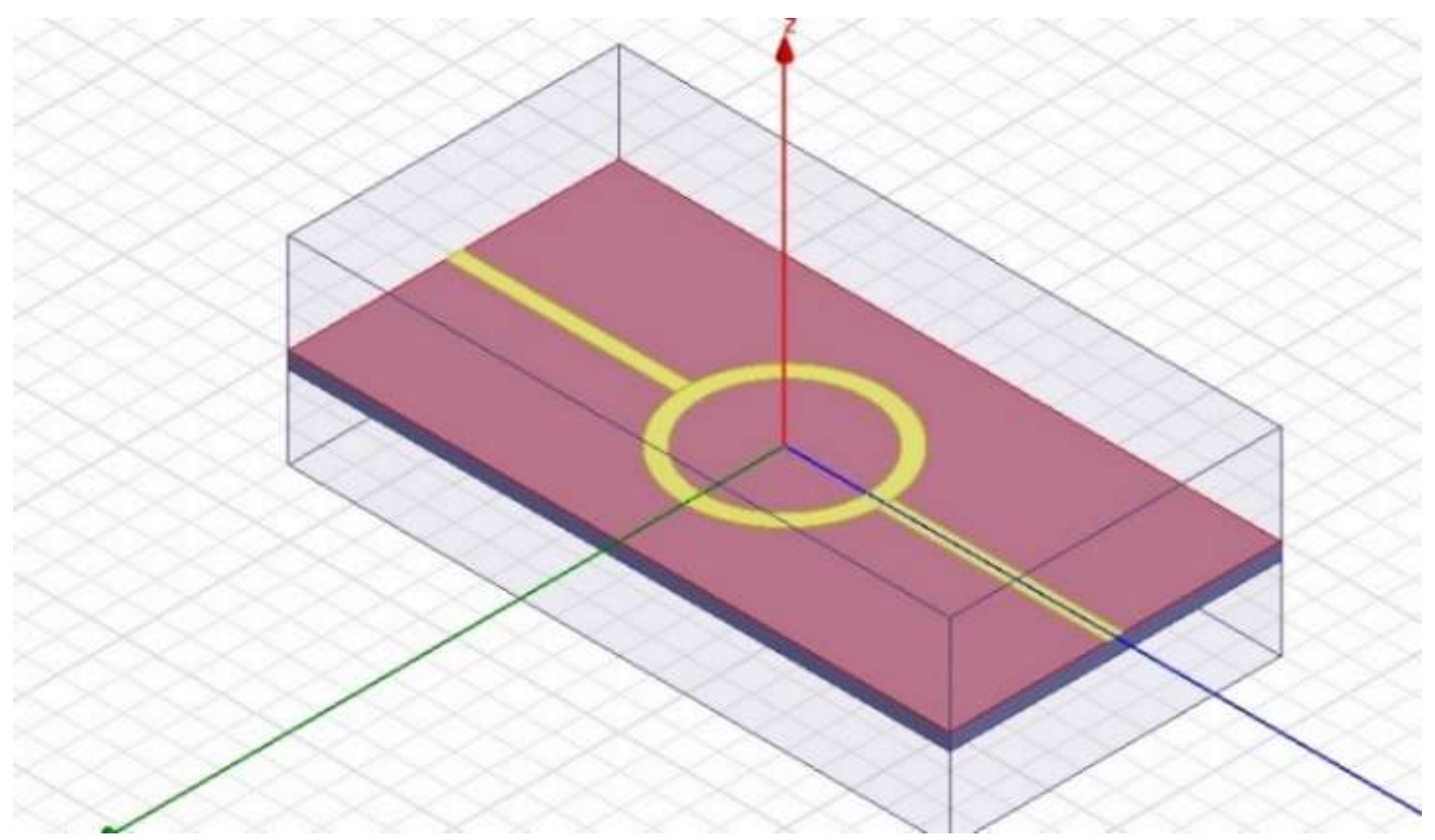

Figure 4:-Destructive Circuit (substrate + thin film) on HFSS Program

The dimension of Ring Resonator we take is shown in the table 2 below:

Table 1:-Dimensions of Ring Resonator

\begin{tabular}{|l|l|}
\hline Parameters & Values \\
\hline Width of feed line & $0.01 \mathrm{~mm}$ \\
\hline Feed line length & $6.95 \mathrm{~mm}$ \\
\hline Coupling gap & $1.52 \mathrm{~mm}$ \\
\hline Total length of substrate & $20 \mathrm{~mm}$ \\
\hline Total width of Substrate & $10 \mathrm{~mm}$ \\
\hline Inner radius of ring & $2.5 \mathrm{~mm}$ \\
\hline Outer radius of ring & $3.035 \mathrm{~mm}$ \\
\hline
\end{tabular}

Variation of film Thickness:-

Varying the thickness of the film for different values of permittivity show all a similar behavior. The resonance frequency seems to shift to the left as the thickness increase, which indicates more effect of the film on the effective permittivity of the system. Here we present a sample. The results in the fig. 5 and fig. 6 below are that of the S11 and S21 parameters for the simulation done taking a permittivity of 100 while varying the thickness of the thin film to see the effect on the result: 


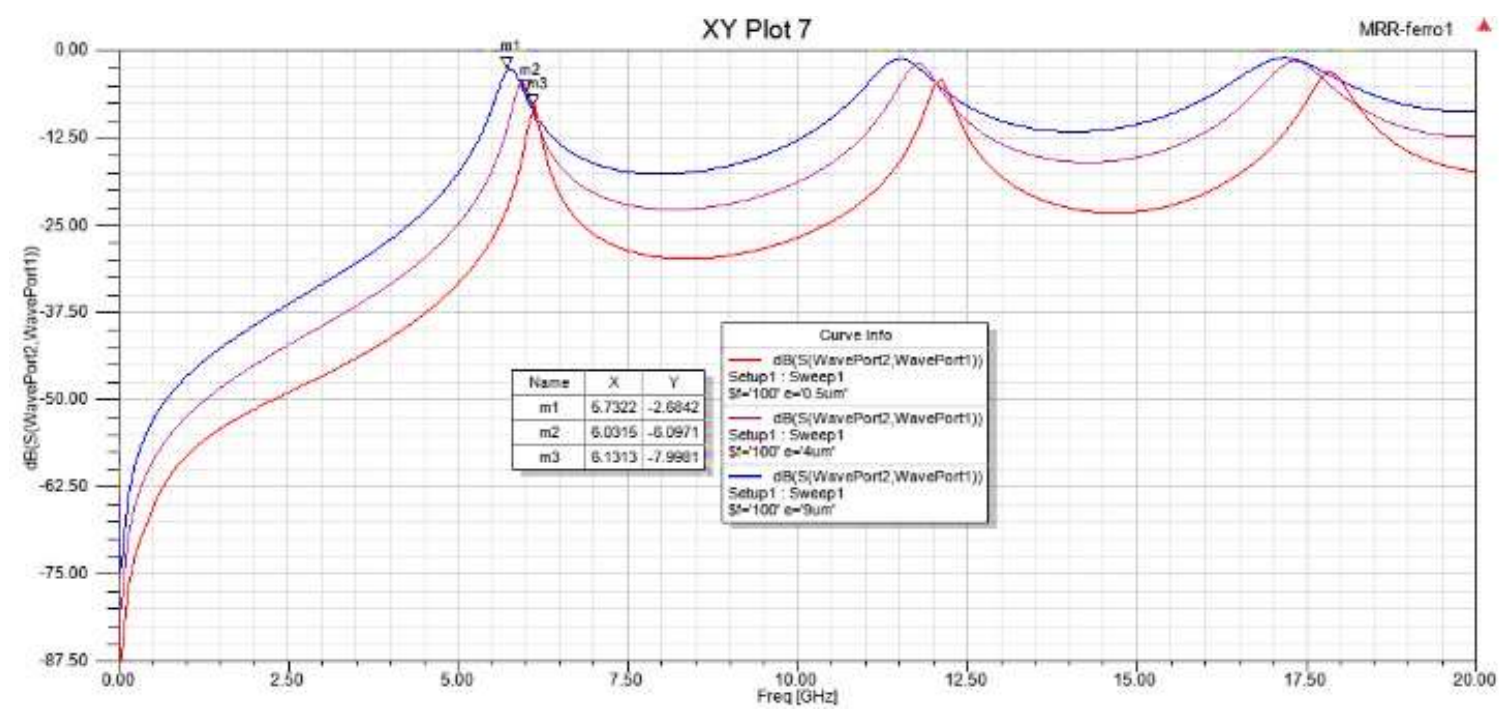

Figure 5:-Results of S21 Parameters at Permittivity 100 at Thickness of (e=0.5, 4, and 9 um)

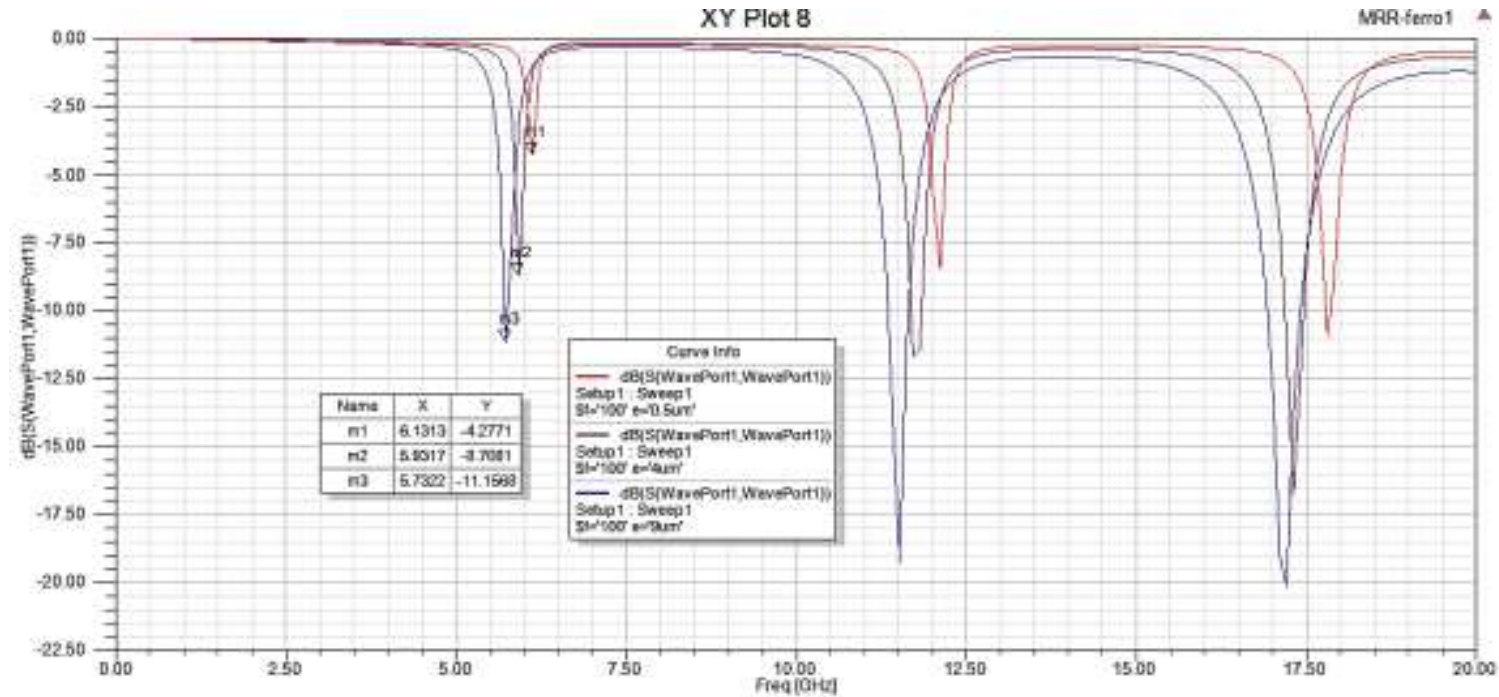

Figure 6:-S11 Parameters at Permittivity 100 at Thickness of ( $\mathrm{e}=0.5$, 4, and 9 um)

Variation of Permittivity ' $\mathcal{E}$ ':-

Varying the permittivity of the film for different values of thickness also show a similar behavior to the of thickness variation. The resonance frequency seems to shift to the left as the thickness increase, which indicates more effect of the film on the effective permittivity of the system. Here we present a sample. The graph results in fig. 7 shows how the resonance is shifted to the left while increasing the permittivity of the film. 


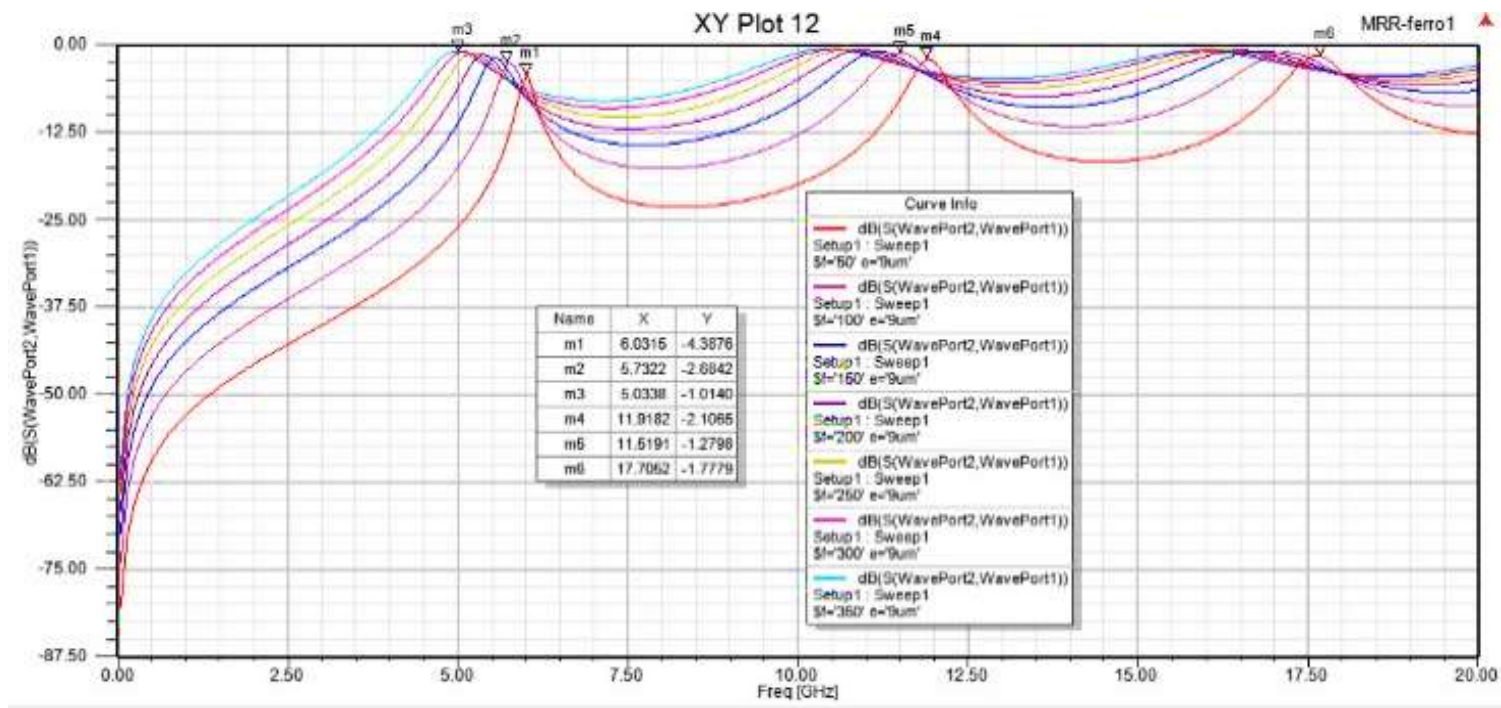

Figure 7:-S21 parameters of permittivity $(50,100,150,200,250,300$, and 350) at thickness e=9um

After many simulations, while varying the thickness and permittivity over a wide range of values, 2 types of plots are done:

1. Permittivity Vs Resonance: The behavior of the permittivity variation Versus resonant frequency for all specified values of thickness are plotted.

2. Thickness Vs Resonance: The behavior of the thickness variation Versus resonant frequency for all specified values of permittivity results are plotted too.

The plots are fitted to a linear curve, and the results seems comparable.

Variation of thin film Permittivity ' $\mathcal{E}$ ':-

Fig. 8 and Fig. 9 below show the variation of permittivity as function of resonance frequency F1 (GHZ) for thicknesses $(\mathrm{e}=6,9 \mathrm{um})$.

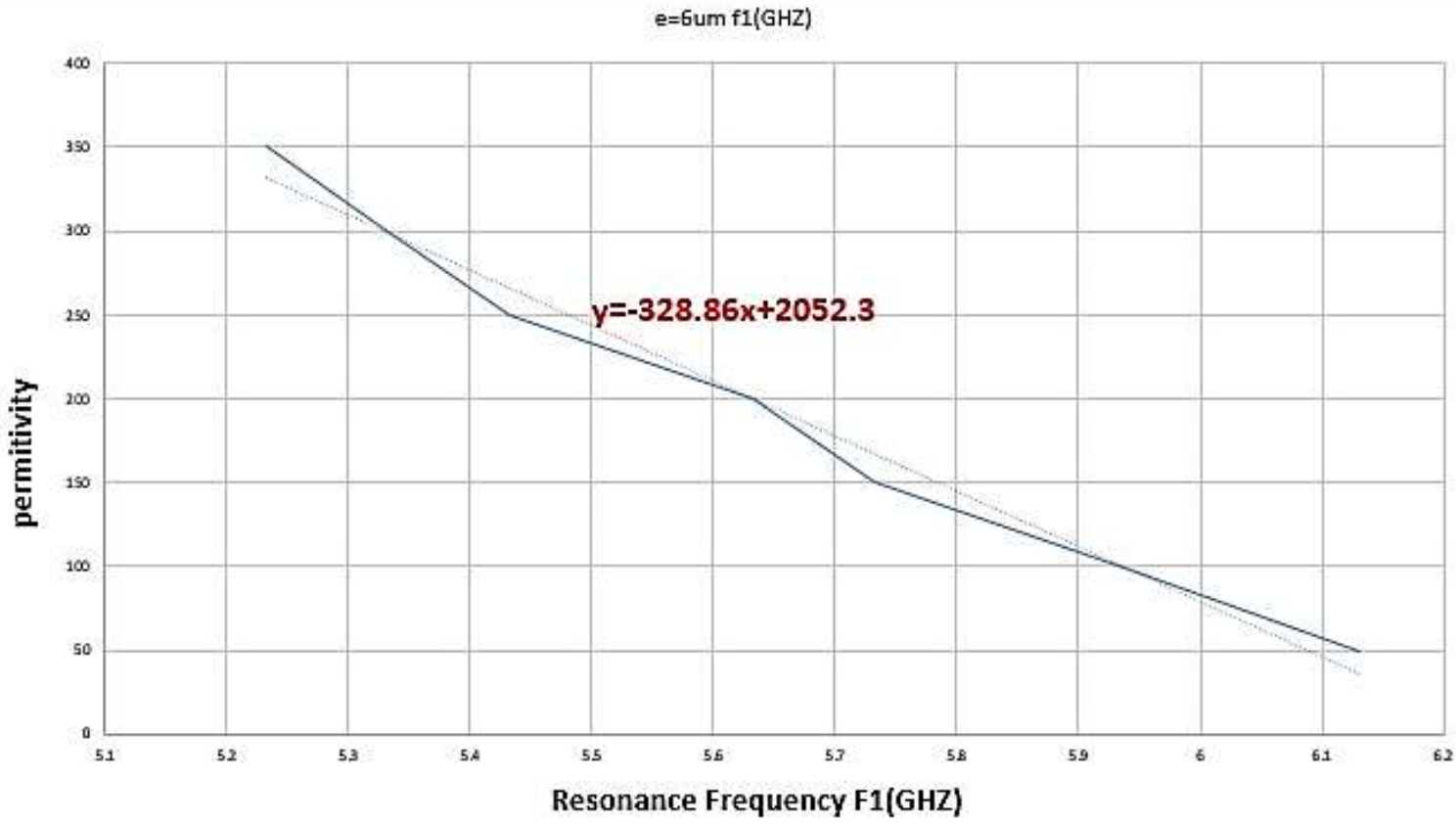

Figure 8:-Result Show the Variation of Permittivity as Function of Resonance Frequency F1 (GHZ) at Thickness $\mathrm{e}=6 \mathrm{um}$ and its Equation 


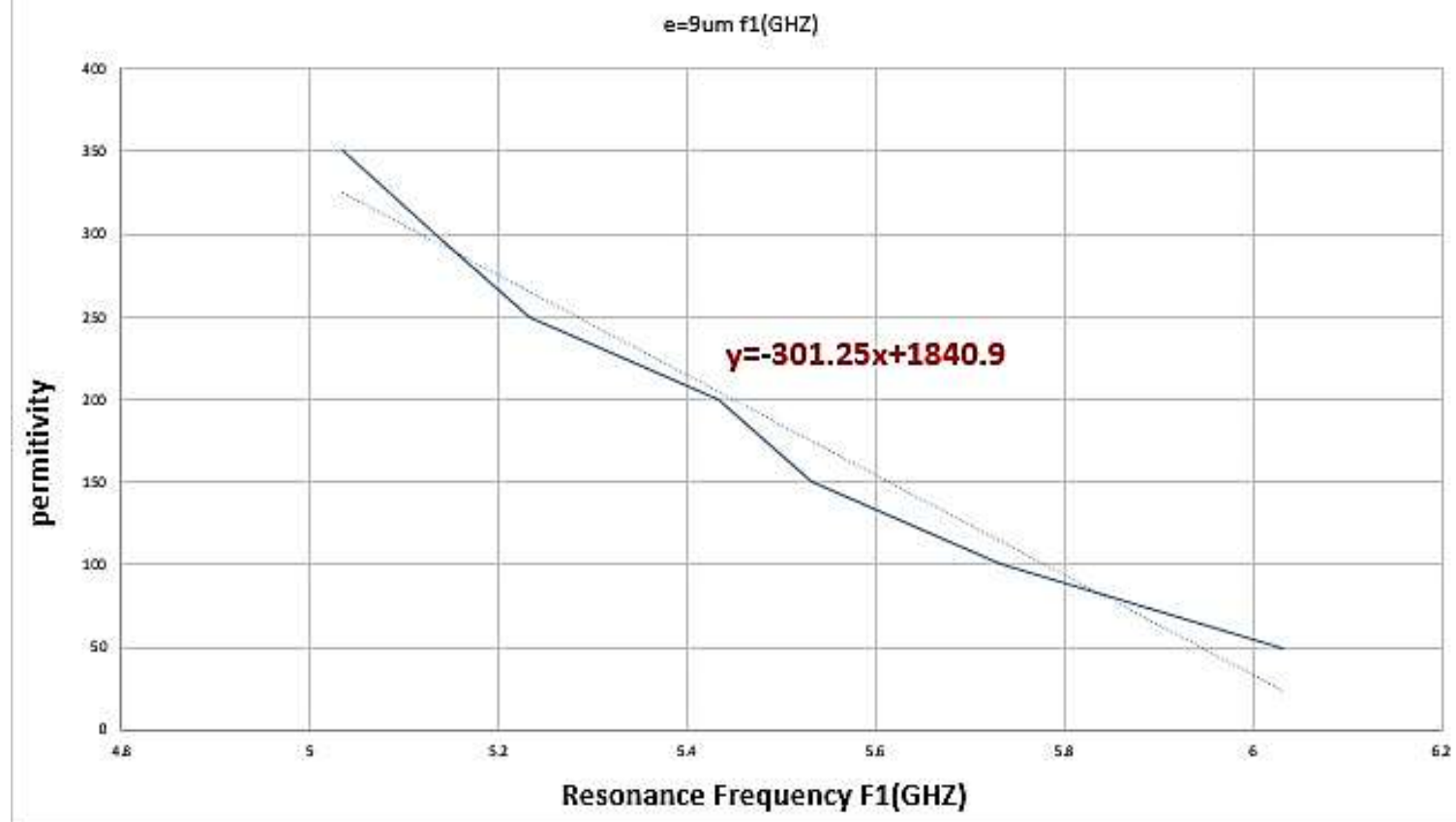

Figure 9:-Result Show the Variation of Permittivity as Function of Resonance Frequency F1 (GHZ) at Thickness $\mathrm{e}=9 \mathrm{um}$ and its Equation

Similar analysis is done for the $2^{\text {nd }}$ and third resonances F2 and F3 as shown in fig. 10 and fig. 11.
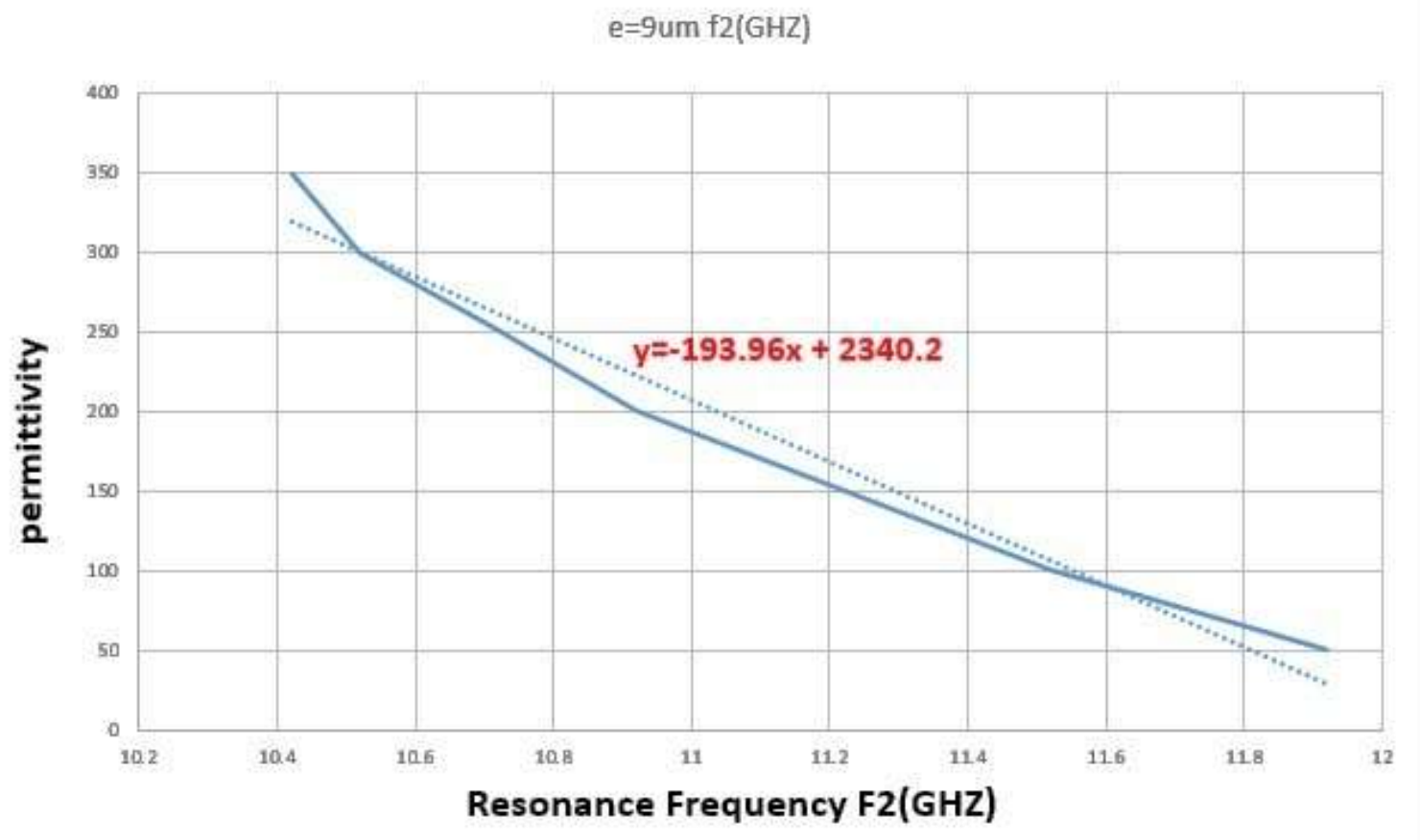

Figure 10:-Result Show the Variation of Permittivity as Function of Resonance Frequency F2 (GHZ) at Thickness $\mathrm{e}=9$ um and its Equation. 


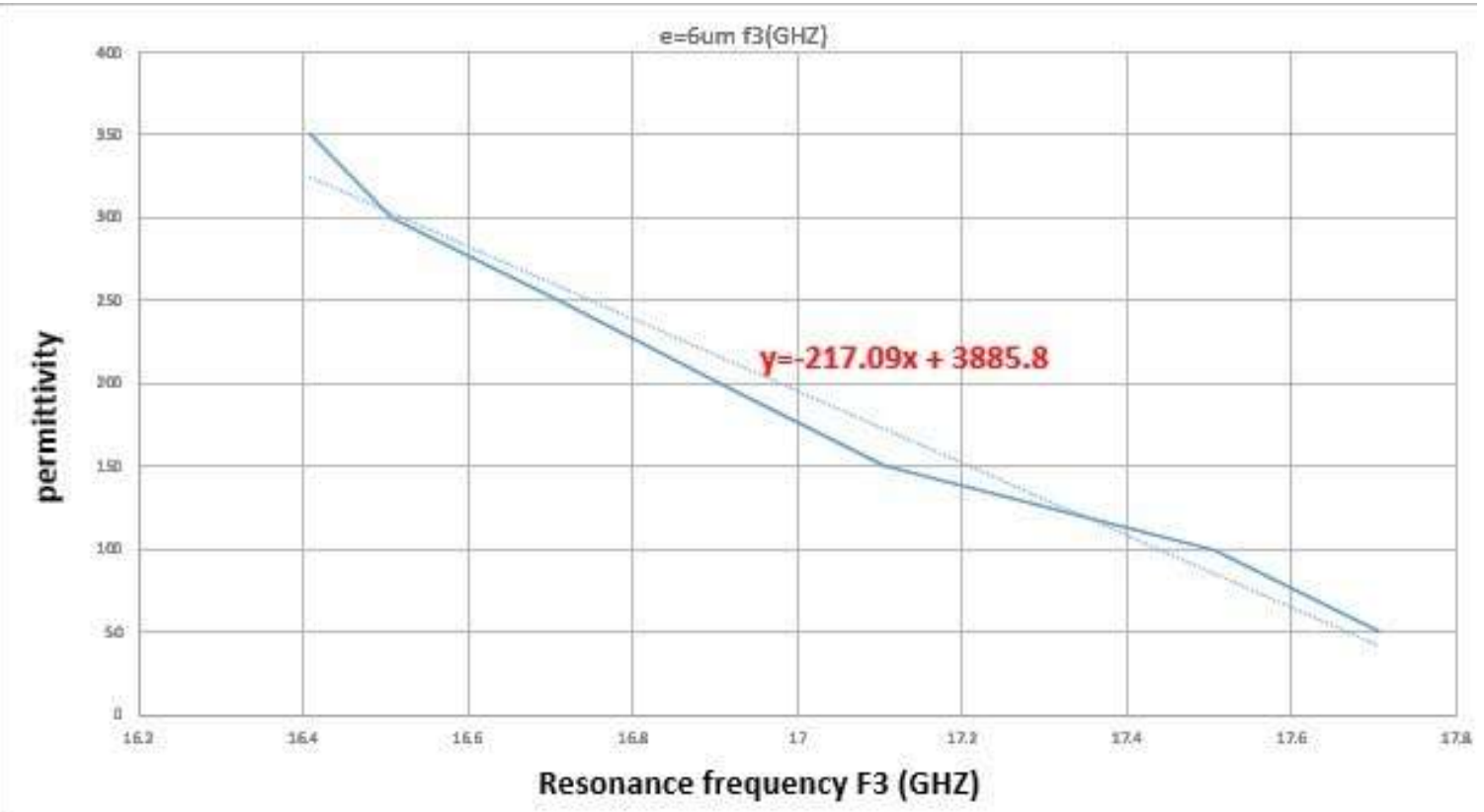

Figure 11:-Result Show us the Variation of Permittivity as Function of Resonance Frequency F3 (GHZ) at Thickness e $=6 u m$ and its Equation

Comparing all the obtained graphs an average linear equation (fig. 12) is found for the variation at the resonance frequency F1:

$$
y=-319.34 x+1967.77
$$

Where $\mathrm{y}$ is the permittivity and $\mathrm{x}$ is the resonance frequency.

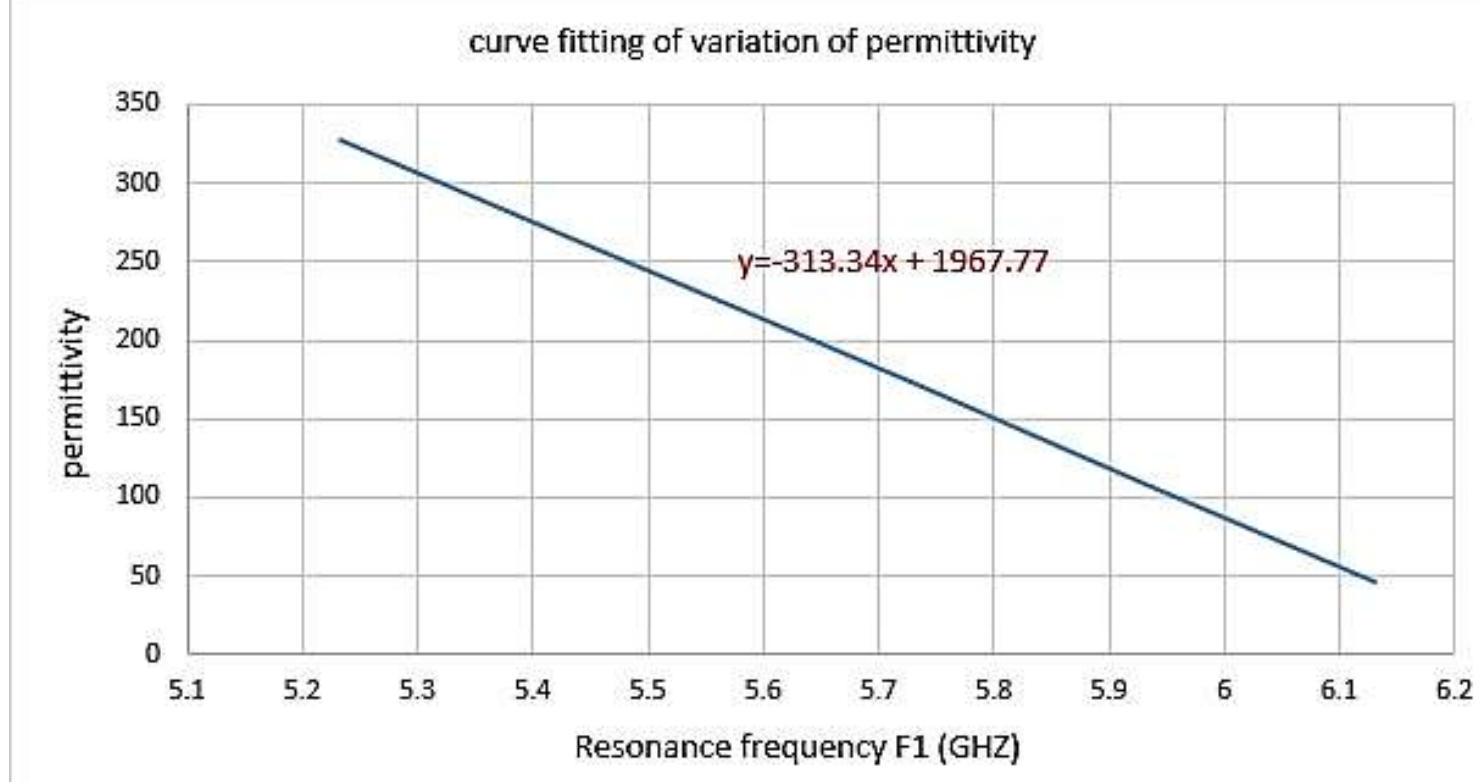

Figure 12:-Variation of Permittivity as Function of Resonance Frequency F1 (GHZ)

The work procedure is conducted as below:

For a sample with known thickness, simulation or measurement is done. And depending on the resonance frequency obtained with the thickness previously specified, the above curve is used to extract the permittivity of the thin film. 
To validate the analysis, a simulation is done, for a thin film material of permittivity $\varepsilon_{\mathrm{r}}=200$ and thickness $\mathrm{e}=6.5$ um.

Assuming the permittivity is unknown, the simulation lead to a resonance frequency of F1=5.532625 GHZ.

Using the above curve whose equation is: $\mathrm{y}=-319.34 \mathrm{x}+1967.77$

The value $y=200.98$ is obtained as the result for the permittivity. This value is not far from the real value. The calculation leads to an error that can be depicted as below:

$$
\text { Error }=\frac{200.98-200}{200}=+0.49 \%
$$

An error of $0.5 \%$ is obtained for this test and similar error for other examples.

Variation of Thickness 'e':-

A similar procedure is followed for the thickness versus resonance frequency; and a curve fitting is done for each thickness variation at a specified permittivity.

permitivity=200 f1(GHZ)

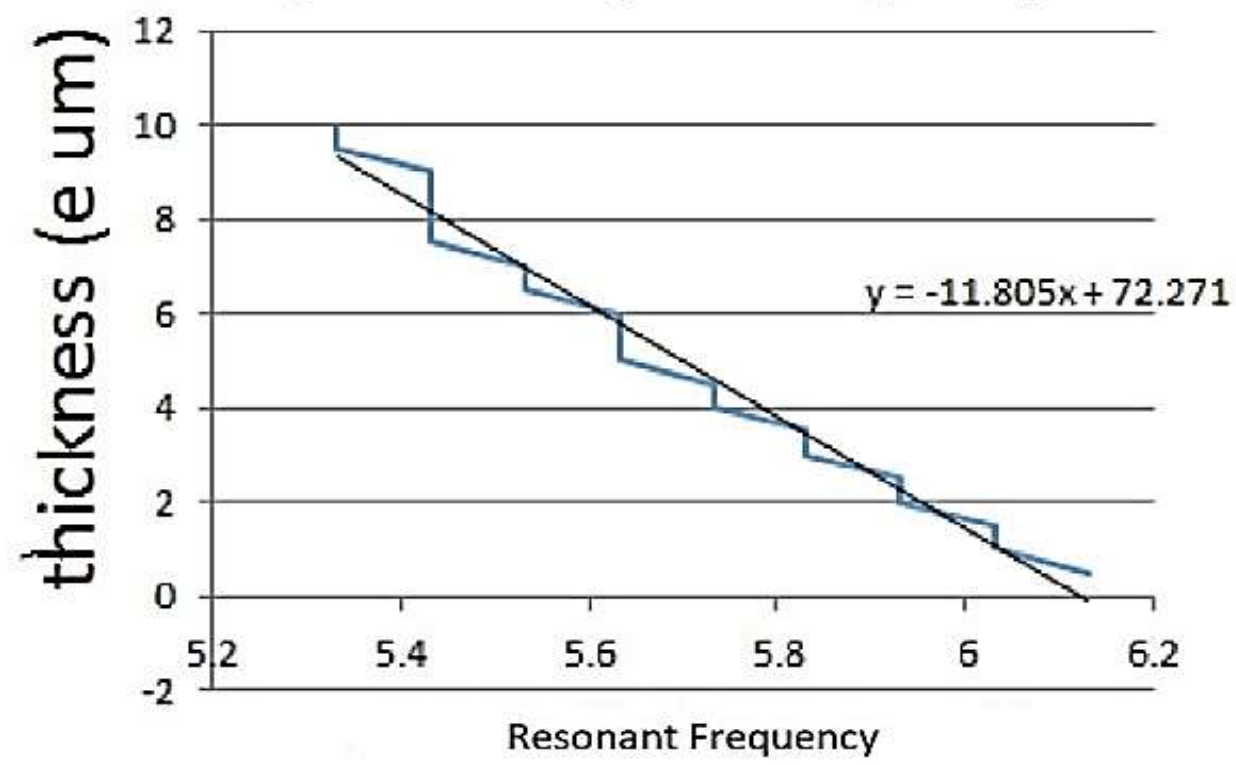

Figure 13:-Result Show us the Variation of Thickness as Function of Resonance Frequency F1 (GHZ) at Permittivity $\in=\mathbf{2 0 0}$ and its Equation 


\section{permitivity=300 f1(GHZ)}

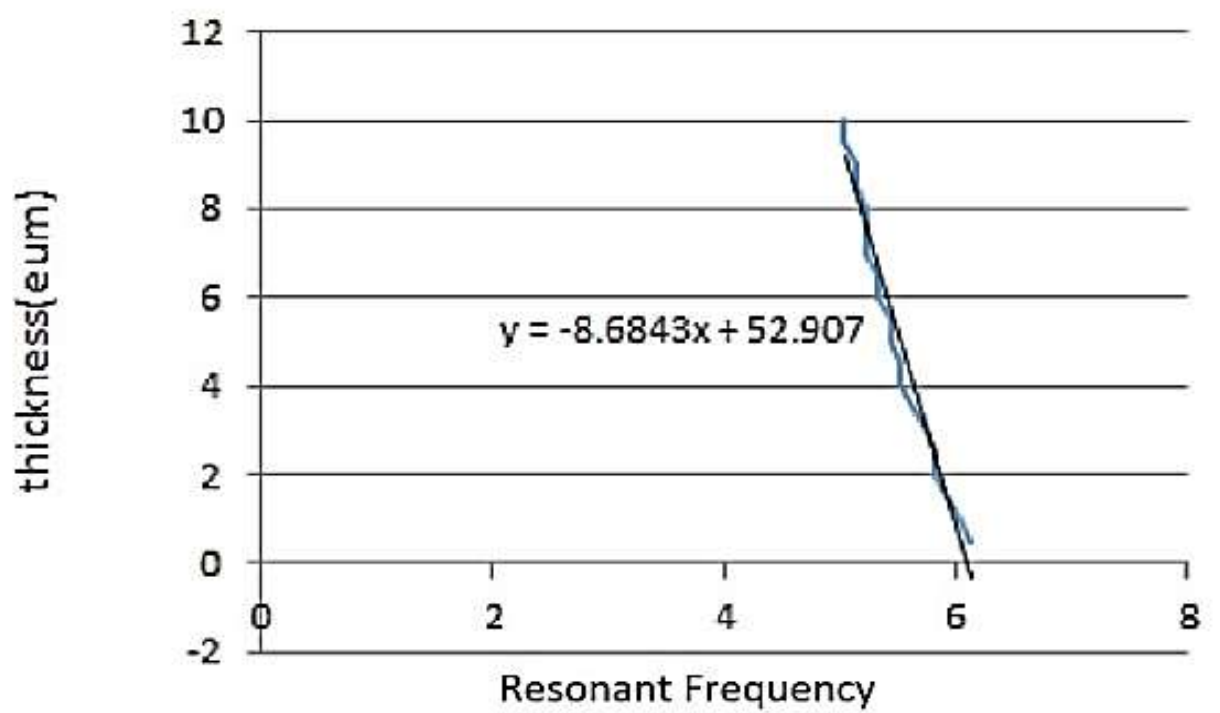

Figure 14:-Result Show us the Variation of Thickness as Function of Resonance Frequency F1 (GHZ) at Permittivity $\epsilon=\mathbf{3 0 0}$ and its Equation

Fig. 13 \& Fig. 14 above show the variation of thickness as function of resonance frequency F1 (GHZ) at permittivity $\varepsilon=200,300$. And similar analysis can be done for frequencies F2 and F3.

Comparing all the obtained graphs an average linear equation (fig. 15) is found for the variation at the resonance frequency F1:

$$
y=-10.51932 x+64.3814
$$

Where $\mathrm{y}$ is the Thickness and $\mathrm{x}$ is the resonance frequency.

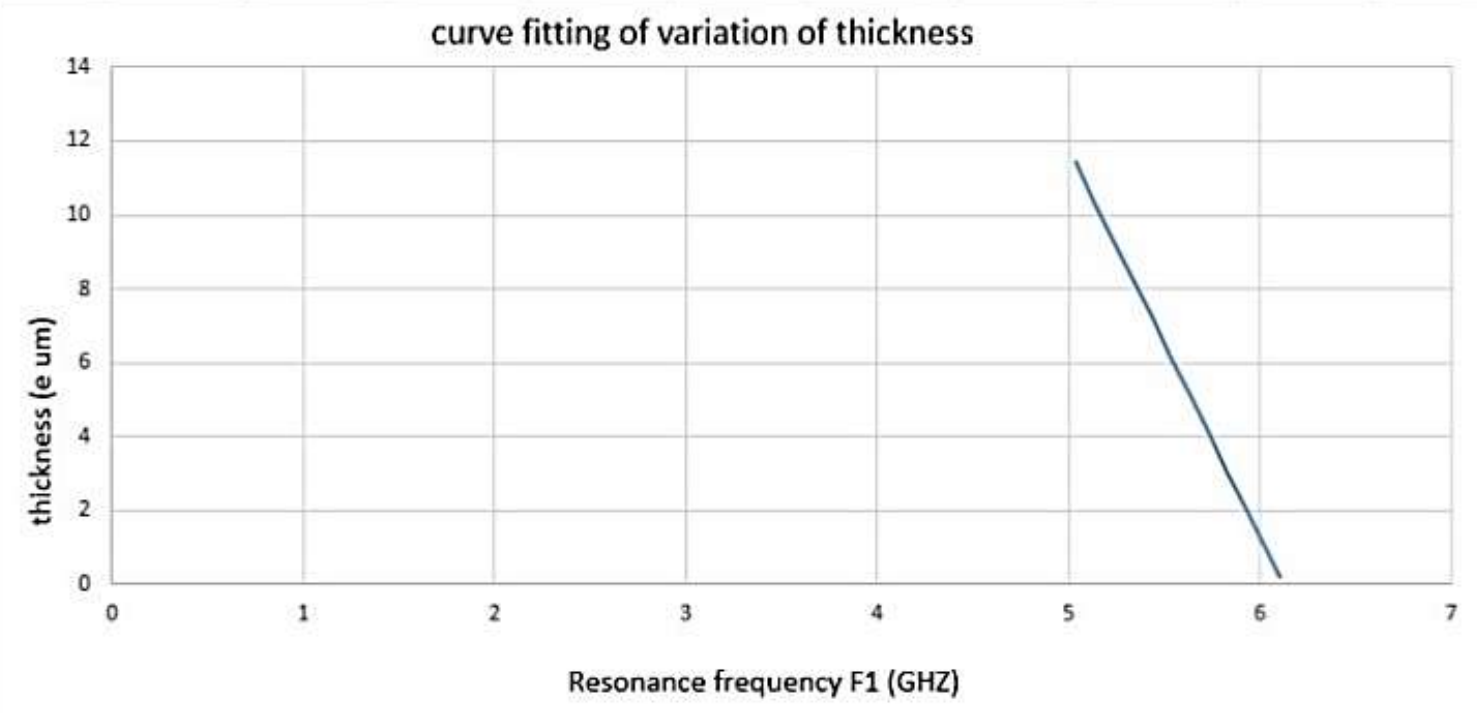

Figure 15:-variation of thickness as function of Resonance frequency F1 (GHZ) 
The work procedure is conducted in a similar manner to before, taking sample with known permittivity, simulation or measurement is done. And relying on the value of the resonance frequency obtained with the permittivity previously specified, the above curve is used to extract the thickness of the thin film.

To validate the analysis, a simulation is done, for a thin film material of permittivity $\varepsilon_{\mathrm{r}}=200$ and thickness $\mathrm{e}=3$ um.

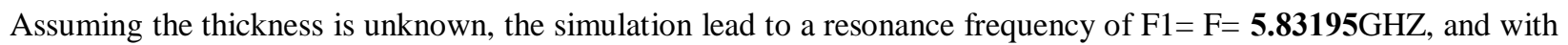
the thickness variation equation : $\mathrm{y}=-10.51932 \mathrm{x}+64.3814, \mathrm{y}=3.033$ is obtained from calculation

Also a small error Error $=\frac{3.033-3}{3}=1.1 \%$ is obtained from thickness extraction.

Although the calculations are done with high performance commercial Electromagnetic software simulator, testing the method with real measurements with prototype will be very interesting. The simulations results are perfect, but with real life measurements more accurate results are achieved.

\section{Conclusion:-}

Simple planar ring resonator is outlined and applied. Simulation results on HFSS are obtained. The method used is an equation based one, that can be used for the MRR sensor of the dimensions specified in the text. Good results are obtained for the permittivity of thin film with an error less than $1 \%$.

The method lacks the real measurement and the dielectric losses measurements described by the loss tangent, which is another important parameter to measure for dielectric materials.

In this research, and in order to get more precise results i.e. a more exact equation representing the variations, more simulation should be done and on a larger range of frequency. The fact that, the simulation time for each step (thickness or permittivity variation) takes very long time (12 hours), has limited the number of steps.

Also, another idea to get higher precision is to switch to more complex form of equation (polynomial and exponential expressions).

\section{References:-}

1. Kumar, Sam Zhang, Lin Li, Ashok. "Materials characterization techniques". Boca Raton: CRC Press. ISBN 1420042947 (2009).

2. Yaw, Kuek Chee. "Measurement of dielectric material properties." Application Note. Rohde \& Schwarz (2012).

3. Zivkovic, Irena, and Axel Murk. "Free-Space Transmission Method for the characterization of dielectric and magnetic materials at microwave frequencies", microwave materials characterization, 2012.

4. Hussein Kassem, Valerie Vigneras, "Non Destructive Dielectric Characterization of thin ferroelectric Films Materials Using Coplanar Line Structure", Integrated Ferroelectrics, 10/2007.

5. D. Thompson, M. Falah, X.Fang and D. Linton - dielectric characterization using the microstrip resonator method.

6. T.C. Edward and M.B.Steer: "Foundations of Interconnect and Microstrip Design "John Wily and Sons, Ltd, 3rd ed., 2000.

7. Isaac Waldron - ring resonator method for dielectric permittivity measurement of foams.

8. Garg Bahl - micro strip lines and slot line. 